\title{
Cancer and COVID-19: Why are cancer patients more susceptible to COVID-19?
}

\author{
Saptarshi Sinha ${ }^{1}\left[\right.$ Chanakya Nath Kundu ${ }^{1}$
}

Received: 21 June 2021 / Accepted: 20 July 2021 / Published online: 24 July 2021

○) Springer Science+Business Media, LLC, part of Springer Nature 2021

\begin{abstract}
The COVID-19 pandemic has engulfed the entire world and has claimed more than 3 million lives worldwide. This viral disease is caused by severe acute respiratory syndrome coronavirus-2 (SARS-CoV-2) and mainly characterized by fever, dry cough, fatigue, anosmia, anorexia, and dyspnea. The severity of the disease increases with age and presence of comorbidities, including cancer. Multiple clinical studies have shown that the cancer patients are highly susceptible to the severe form of the viral disease. In this review article, we have summarized the available scientific literature regarding the molecular links between COVID-19 and cancer, which make the cancer patients highly susceptible to COVID-19. Few studies have shown that the angiotensin-converting enzyme 2 (ACE2) receptor, transmembrane protease serine 2 (TMPRSS2), and the immune response and inflammation establish the interconnection between the two diseases. Additionally, we have also discussed whether SARS-CoV-2 can contribute to cancer development in COVID-19 patients. A recent study has suggested that SARSCoV-2 may create a microenvironment that may support cancer cell proliferation and induce the activation of dormant cancer cells (DCCs). In another study, the blood sera of COVID-19 patients were found to activate epithelial-to-mesenchymal transition (EMT) in cancer cells. Overall, this review article will surely help the scientific community to understand why the cancer patients are so much prone to COVID-19 and will also motivate the researchers to find new therapeutic strategies that may save the lives of many COVID-19-infected cancer patients.
\end{abstract}

Keywords COVID-19 $\cdot$ Cancer $\cdot$ Immune response $\cdot$ Inflammation $\cdot$ Blood coagulation $\cdot$ Oncovirus

\section{Introduction}

Since the end of 2019, mankind is combating a global health crisis due to the outbreak of COVID-19 disease, which is caused by severe acute respiratory syndrome coronavirus- 2 (SARS-CoV-2) [1]. It is a single-stranded RNA virus. The virion is spherical in shape and its surface is covered by spike-like projections, which give the virus the structure of a "solar corona" [2]. In December 2019, a number of respiratory distress cases were first reported in Wuhan, China [3]. Since then, COVID-19 has affected multiple countries, such as India, the United States of America, France, Brazil, Turkey, Russian Federation, the United Kingdom, Italy,

Chanakya Nath Kundu

cnkundu@kiitbiotech.ac.in

1 Cancer Biology Division, School of Biotechnology, Kalinga Institute of Industrial Technology, KIIT Deemed to be University, Campus-11, Patia, Bhubaneswar, Odisha 751024, India
Argentina, Germany, Spain, and Colombia [4]. On January 31, 2020, COVID-19 was declared by the World Health Organization (WHO) as a "Public Health Emergency of International Concern (PHEIC)" [5] and on March 11, 2020, this disease outbreak was characterized as a "pandemic" [6]. As on June 14, 2021, according to the WHO, the number of confirmed cases worldwide has exceeded 175 million with the number of global deaths more than 3.7 million [7].

SARS-CoV-2 is primarily transmitted via direct contact or through respiratory droplets spread by coughing or sneezing from a COVID-19 patient [8]. SARS-CoV-2 infection requires a median period of incubation of around 4-5 days before the disease symptoms start showing up. At the onset of the sickness, the patients typically experience fever, dry cough, anosmia, anorexia, and fatigue. With time, they start to experience dyspnea, arthralgia, headache, diarrhea, nausea, and hemoptysis. A chest CT scan of a COVID-19 patient showed signs of pneumonia along with other complications, such as RNAemia, acute cardiac injury, acute respiratory distress syndrome (ARDS), and secondary infections [9]. 
ARDS sets in after 8-9 days from symptom onset and is characterized by dyspnea and hypoxemia. It may also lead to respiratory failure. The airways of a COVID-19 patient get so much damaged that it attracts aggressive inflammatory responses. The immune system enhances the production of cytokines, resulting in a cytokine storm and sepsis. This hyper-inflammatory response inflicts serious damage to the organs, leading to a multi-organ failure [10].

During the ongoing pandemic, the COVID-19 disease has proved to be detrimental to the health of the cancer patients. The cancer patients are reported to be highly susceptible to the severe symptoms of the viral disease. Recently, a study was performed with 536 non-cancer patients and 105 cancer patients, who were of same age and infected with COVID19. It was observed that the cancer patients showed all the severe symptoms of COVID-19. The severe symptoms were mainly observed in the patients suffering from hematological, lung, and stage IV cancers. Metastatic cancer patients infected with COVID-19 exhibited a higher death risk [11]. Another recent study showed that 18 patients, out of 1590 COVID-19 positive cases, had a history of cancer. The most common type of cancer in this study was found to be lung cancer. The cancer patients were much older (mean age 63.1 years) than the other patients (mean age 48.7 years). They deteriorated very fast (median time 13 days) in comparison to 43 days in the case of non-cancer patients [12]. Desai et al. showed that there was a $2 \%$ prevalence of cancer in COVID-19 patients and the cancer patients, who had a surgery or received chemotherapy in 1-month time, had a major risk (75\%) of developing the severe COVID-19 complications than the non-cancer COVID patients (43\%) [13]. Zhang et al. showed that 15 out of 28 (53.6\%) COVID19-infected cancer patients developed severe COVID symptoms and 8 patients (28.6\%) died. The most common type of cancers in the study were found to be lung, esophageal, and breast cancers. The cancer patients, who received anticancer treatments within 14 days prior to COVID-19 diagnosis, developed the severe clinical symptoms in comparison to those who did not receive any anti-cancer treatment during the said period [14]. Mehta et al. carried out another study with 218 COVID-19-positive cancer patients, of which 61 (28\%) patients died due to the COVID-19 infection. Mortality rates were very high in patients suffering from lung (55\%), gastrointestinal [upper GI (38\%), pancreatic (67\%), colorectal (38\%)], and gynecological (38\%) cancers [15]. In a recent study, out of 1524 cancer patients, 228 (14.96\%) patients were reported to suffer from non-small cell lung cancer (NSCLC). The NSCLC patients, who were older than 60 years of age (4.3\%), were found to be more susceptible to COVID-19 than those aged 60 years or less (1.8\%) [16]. Another study showed that the stage IV oral cancer patients, who received anti-cancer treatments and were infected with COVID-19, had the worst effects and highest death rate than all those patients who did not contract COVID-19 after the anti-cancer treatments [17]. The above studies conducted with COVID-19-infected cancer patients clearly showed that they were more susceptible to COVID-19 and subsequently developed more severe clinical symptoms and deteriorated very fast. Hence, it is imperative to understand why the cancer patients are so much prone to this deadly viral disease.

In the present review, we have discussed briefly the molecular links between cancer and COVID-19 and the clinical symptoms that the cancer patients develop after getting infected with COVID-19 (Fig. 1). We have also talked about whether SARS-CoV-2 possesses any tumorigenic activity that can cause cancer in patients after recovery from COVID-19.

\section{COVID-19 and cancer: Connecting links between the two diseases}

In COVID-19 disease, after entering the human body, SARS-CoV-2 binds to the angiotensin-converting enzyme 2 (ACE2) receptor present on the surface of the host cells [18]. ACE2 receptors are present in multiple tissues, including cornea, nose, lungs, esophagus, heart, stomach, liver, ileum, colon, gall bladder, kidney, and testis [19]. The virus attaches to the ACE2 receptor with the help of the spike (S) proteins, which are cleaved by host proteases, such as transmembrane protease serine 2 (TMPRSS2), cathepsin $\mathrm{L}$, and furin. Cleavage of the $\mathrm{S}$ proteins help in membrane fusion that results in the release of viral RNA into host cell cytosol [20-22].

Recently, the importance of ACE2 receptors in the pathogenicity of the virus was examined by infecting the human

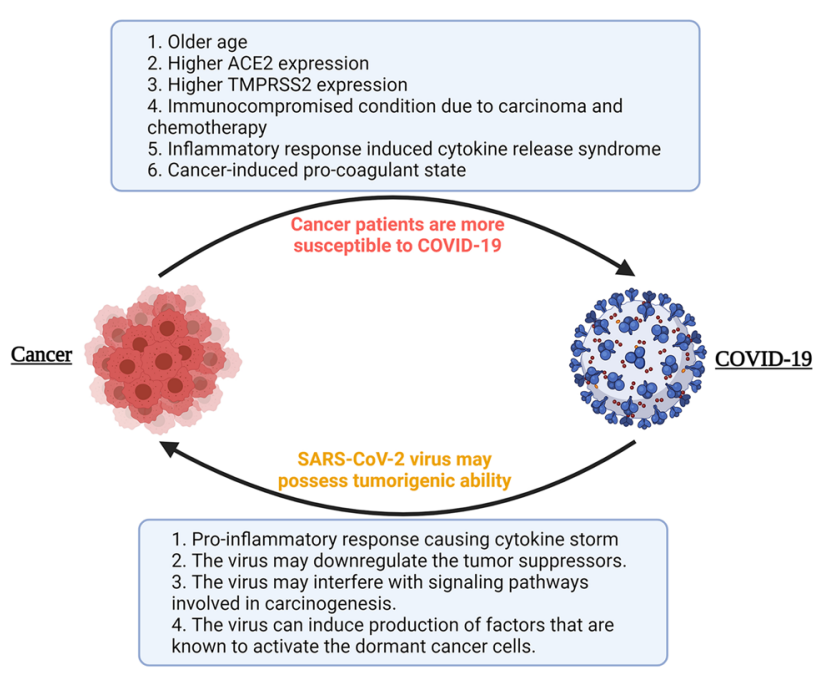

Fig. 1 Inter-connection between COVID-19 and Cancer. Created with www.BioRender.com 
ACE2-bearing transgenic mice with the virus. The infected ACE2 mice experienced weight loss and virus replication in the lungs, along with incidence of interstitial pneumonia and invasion of lymphocytes and macrophages into the alveolar interstitium, and macrophage build-up in the alveolar cavities. The bronchial and alveolar epithelial cells and macrophages showed the presence of the viral antigens. On the other hand, the wild-type mice did not develop the viral infection, confirming pathogenicity of the virus in human ACE2-bearing mice [23]. In a recent study, it has been observed that the expression of ACE2 in the lungs increases with age [24]. It has also been noticed that the older individuals, who are more than 60 years of age, and/or with a compromised immune system are highly susceptible to COVID-19 [25]. Since cancer is more common in the older population [26] and ACE2 expression increases with increasing age, the cancer patients have a greater chance of experiencing the severe symptoms when infected with SARS-CoV-2 [27]. Moreover, ACE2 expression is also found to be elevated in the lungs of patients who are regular smokers and those who suffer from smoking-related lung diseases, including lung cancer [28]. Hence, smokers and the patients suffering from lung cancer have a higher risk of getting infected with the severe form of the COVID-19 disease.

Another link between COVID-19 and cancer involves the host protease TMPRSS2, which is required for SARSCoV-2 to enter the host cells and release the viral RNA. This protease is an androgen-regulated gene and found to be highly upregulated in prostate cancer. The androgen receptor is present in both lung and prostate cells and thus may have a crucial role to play in the expression of TMPRSS2 in the lung and prostate tissues [29]. A recent study has shown that when prostate cancer patients were subjected to androgen deprivation therapy (ADT), there was a significant reduction in COVID-19 infection in comparison to the patients without ADT or suffering from other types of cancer [30]. Another study showed that the prostate cancer patients subjected to ADT were associated with reduced rates of hospitalization and need for oxygen supplementation, in comparison to those treated without ADT [31].

\section{Host immune response in COVID-19 and cancer}

It is well known that in comparison to normal and healthy people, the cancer patients are more susceptible to infections because their immune system is suppressed due to the carcinoma and anticancer therapies. Patients, who are suffering from multiple myeloma, leukemia, lymphoma, or other hematological cancers; patients, who have undergone radiotherapy, chemotherapy, or immunotherapy; and all those patients, who have recently undergone a bone marrow or stem cell transplant, will have a greater chance of getting infected with COVID-19 [32]. Macrophages are known to play important roles in the inflammatory responses associated with both cancer and COVID-19. In COVID-19 disease, it has been observed that the M1 macrophages are activated, which are associated with macrophage-activating syndrome (MAS), cytokine storm, lymphopenia, damage of the endothelium, and increase in intravascular blood coagulation. On the other hand, in cancer, M2 macrophages are activated that suppress the immune response and also simultaneously help in tumor progression. As a result of immunosuppression, the antiviral immune response is impaired that makes the cancer patients more susceptible to viral infections [33].

When SARS-CoV-2 induces acute injury in the lung tissues, it results in high levels of pyroptosis, which is an inflammatory, caspase-1-dependent-programmed cell death. Pyroptosis triggers an inflammatory response and induces the release of the cytokines IL- $1 \beta$ and IL-18, which are increased in SARS-CoV-2 infection. During pyroptosis, certain molecular motifs are released from the damaged or dying host cells. These are known as the pathogen-associated molecular patterns (PAMPs) and damage-associated molecular patterns (DAMPs). The viral RNA serves as a PAMP, while ATP, host nucleic acid fragments, and oligomers of apoptosis-associated speck-like protein containing a caspase-recruitment domain (ASC) are typical examples of DAMPs. These molecular motifs are detected by neighboring lung epithelial cells, alveolar macrophages, and vascular endothelial cells, triggering the generation of chemokines and pro-inflammatory cytokines [34]. These cytokines initiate an immune response through the activation of $\mathrm{T}$ helper 1 (TH1) cells, which in turn activates production of more macrophages, monocytes, and $T$ cells at the site of infection. This results in further enhancement of the inflammatory response, thereby creating an inflammatory feedback mechanism. This buildup of immune cells within the lungs causes a higher production of pro-inflammatory cytokines, resulting in an overaggressive immune response called "cytokine storm" that can inflict serious damage to the lungs [10,35]. Now, in some types of cancers, the patients are subjected to immunotherapies such as chimeric antigen receptor (CAR) T-cell therapies, and immune checkpoint inhibitors (ICIs). It is well known that these cancer patients develop an acute inflammatory condition called the cytokine release syndrome. It is a similar clinical condition like the cytokine storm that is seen in COVID-19 patients. Thus, when the cancer patients, who are treated with immunotherapies, are infected with COVID-19, the viral infection can intensify the cytokine storm in the patients [36]. This will increase the chances for the cancer patients to develop the severe clinical symptoms of COVID-19 if the concentration of 
the pro-inflammatory cytokines is not controlled during the course of the disease.

The COVID-19 patients experience the cytokine storm, which forms an interplay between inflammation and coagulation. During an immune response, the overproduction of pro-inflammatory cytokines helps in the activation of coagulation and intravascular coagulation pathways, which is a hallmark of multi-organ injury in sepsis [37]. The inflammation-induced coagulation is initiated by the tissue factor (TF) pathway. As a response to pro-inflammatory cytokines, TF is expressed on both mononuclear cells, such as monocytes and vascular endothelial cells. It promotes the conversion of prothrombin to thrombin, which is a central player in blood coagulation. Thrombin initiates the process of blood clotting through the activation of platelets and conversion of fibrinogen to fibrin [38]. This promotes the development of microthrombosis, disseminated intravascular coagulation (DIC), and multi-organ failure, which are commonly seen in severe COVID-19 cases [34]. Venous thromboembolic events (VTEs) have been found to be a common complication in cancer patients. They are at a higher risk of developing thrombotic events than a healthy individual. The cancer cells increase the production of coagulation factors and proinflammatory cytokines. This results in activation of blood coagulation pathways, which aids in the development of VTEs. The factors that can elevate the chances for the cancer patients to suffer thrombotic complications include old age, immobility, the type and stage of cancer, and chemotherapy and surgery [36]. In a recent study, venous thromboembolisms were evaluated in COVID-19 patients. The intensive care unit (ICU) patients showed around $9.3 \%$ of symptomatic VTEs. The symptoms of VTEs were developed during the course of the disease and can be used as an indicator for the severity of COVID-19 [39]. Further clinical studies are required to prove that VTEs can increase the mortality rates in cancer patients infected with COVID-19.

\section{Can SARS-CoV-2 cause cancer?}

We have discussed before that SARS-CoV-2 causes inflammation like many other oncoviruses. But there is no evidence whether the virus possesses any tumorigenic activity. When a host system is infected with an oncovirus, the levels of proinflammatory cytokines (IL-6) are highly elevated, which is also a common event in COVID-19 disease. In COVID-19 patients, the IL-6 levels are found to be highly upregulated, which activate the inflammatory signaling pathways, resulting in an induction of cytokine storm [40]. This gives an indication that SARS-CoV-2 may possess cancer-causing ability. Previously, it was reported that the coronavirus endoribonuclease Nsp15 interacts with the retinoblastoma protein, which is a tumor suppressor. As a result of this interaction, the retinoblastoma protein is downregulated, resulting in alteration of gene expression and enhancement of cell division and growth [41].

SARS-CoV-1 is the predecessor of SARS-CoV-2. SARS$\mathrm{CoV}-1$, which caused the SARS disease, was found to interfere with the signaling pathways that play important roles during carcinogenesis, including MAPK, p53, JAK/STAT, and EGFR signaling pathways. SARS-CoV-1 was found to activate p38 MAPK that activates the production of the proinflammatory cytokines. It was also found to interact with an E3-ubiquitin ligase, RCHY1. This interaction caused enhanced degradation of the tumor suppressor protein, p53 [42]. Post-SARS-CoV-1 infection, the EGFR signaling pathway was found to be highly upregulated, which resulted in an advanced lung tissue damage and deregulated wound repair mechanisms. This caused pulmonary fibrosis in the SARS patients [43]. Since fibrosis has been previously shown to be a risk factor for carcinogenesis [44], this also indicates that SARS-CoV-1 infection might possess tumorigenic activity. Since SARS-CoV-1 and SARS-CoV-2 both belong to the same family of coronaviruses, it is highly important to examine whether SARS-CoV-2 can induce carcinogenesis in COVID-19 patients.

A recent study has suggested that SARS-CoV-2 may help in the generation of a microenvironment that supports proliferation of cancer cells and may induce production of factors that are known to activate the dormant cancer cells (DCCs). In COVID-19 disease, the host immune system shows hyperactivation of macrophages, neutrophils and monocytes, overproduction of pro-inflammatory cytokines, and lymphopenia. The activated neutrophils produce certain factors that induce tissue damage. Such factors include the neutrophil extracellular traps (NETs), which are web-like structures of DNA and proteins. A recent study showed that during autopsy of deceased COVID-19 patients, enhanced infiltration of neutrophils was found in the lungs. Another study confirmed that NETs play an important role in inducing immuno-thrombosis in COVID-19 patients. On the other hand, it has recently been hypothesized that NETs may reactivate DCCs in the COVID-19-induced pro-inflammatory microenvironment, thereby enhancing the chances for cancer relapse and metastasis [45]. During severe COVID-19, the pro-inflammatory cytokines induce the activation of NF- $\kappa \mathrm{B}$ in both immune and non-immune cells. The activated NF- $\mathrm{kB}$ induces the reawakening of dormant breast cancer cells (DBCCs) by activating the proliferation of the breast cancer cells and creating a microenvironment favorable for metastasis. Hypoxia is found to be an indicator of poor prognosis in both cancer and severe COVID-19. In breast cancer, hypoxia plays an important role in the activation of genes responsible for dormancy, stemness, epithelial-to-mesenchymal transition (EMT), and drug resistance and helps in the production of DBCCs. Hence, we can say that hypoxia in COVID-19 
patients may induce dormancy of cancer cells and also build up chemoresistance against anti-cancer drugs and therefore may help in cancer relapse [46].

A recent study showed that the expression of ZEBI and $A X L$ mRNA levels was found to be upregulated after SARS$\mathrm{CoV}-2$ infection in lung cancer cells, resulting in induction of EMT. They have also reported that complete inhibition of transcription of the genes, which regulate the functions of tight junction, takes place after the viral infection. Due to higher expression of ZEB1 and TGF- $\beta$, the functions of ACE2 receptor are completely inhibited. All these findings suggest that SARS-CoV-2 infection clearly induces EMT and supports cancer relapse and metastasis [47]. Recently, a group of researchers applied the serum samples of COVID19 patients in the cultures of different cancer cells to examine their proliferation, motility, and gene expression patterns. It was observed that the cancer cells were exhibiting enhanced motility with loss in intercellular connections and the gene expression analysis showed higher expression of ZEB1, SNAIL2, and VIM mRNA levels, all of which indicate activation of EMT [48].

The above discussions are giving a clear indication that coronaviruses, including SARS-CoV-1 and SARS-CoV-2, may possess cancer-causing ability and may promote carcinogenesis. Hence, it is important to carry out multiple research studies to observe whether the coronaviruses have tumorigenic activity.

\section{Conclusion}

In the past 1 year, the COVID-19 pandemic has been wreaking havoc all around the world. It is indeed a sorrowful state for mankind that we are trying to fight a battle with a deadly virus without any weapons to kill the menace. In such a helpless situation, it is very scary to think about the fact that how the doctors are going to manage the cancer patients if they are infected with SARS-CoV-2 virus. This pandemic has affected very badly the healthcare system dealing with cancer diagnosis and treatment. It has been observed that the cancer patients are experiencing the severe form of COVID-19, leading to death of many patients. Symptomatic treatment of the cancer patients, who are affected with COVID-19, has become more and more difficult with subsequent increase in the severity of the viral disease. The molecular links between cancer and COVID-19 have been found to be ACE2, TMPRSS2, pro-inflammatory cytokines, and blood coagulation. These links can be targeted to control the fatal combination of cancer and COVID-19. Some anticancer drugs are being investigated so that they can be repurposed to treat COVID-19. But it is imperative to see that there should be no or minimal drug interactions while the therapies are designed for the treatment of cancer and
COVID-19. These treatment strategies should be able to control the severe effects of COVID-19 and also be useful in the treatment of cancer. The patients, who require anticancer treatments, should be screened for COVID-19 infection before going ahead with the future course of treatments. If COVID-19 is found to be positive in a cancer patient, then the immune-suppressive treatments should be avoided or else the dosages of the treatments can be reduced [49]. It has been recently advised that radiotherapy can be safely continued during the pandemic by decreasing the number of visits to the treatment centers [50]. The oncologists should carefully plan the routine cancer treatments and follow-up appointments so that the cancer patients can benefit from the treatments instead of getting infected by SARS-CoV-2 [51].

It is imperative for the scientific research community to come forward and carry out further studies that will help us learn more about the molecular connections between COVID-19 and cancer, so that the COVID-19-infected cancer patients can be treated effectively without any disruptions in cancer care.

Acknowledgements We are very much thankful to the Indian Council of Medical Research (ICMR), Government of India, for providing a research fellowship (Reference number: 3/2/2/53/2019/Online Onco Fship/NCD-III) to Saptarshi Sinha.

Author contributions SS conceptualized and wrote the original draft of the manuscript. CNK critically reviewed the manuscript and approved the final submitted version.

Funding This research did not receive any specific grant from funding agencies in the public, commercial, or not-for-profit sectors.

Data availability All the information mentioned in this review article are available on the internet.

\section{Declarations}

Conflict of interest The authors declare that they have no competing interests.

Ethics approval Ethical approval was not required for this review article.

\section{References}

1. Zhu N, Zhang D, Wang W, Li X, Yang B, Song J, et al. A novel coronavirus from patients with pneumonia in China, 2019. N Engl J Med. 2020;382(8):727-33. https://doi.org/10.1056/NEJMo a2001017.

2. Singhal T. A review of coronavirus disease-2019 (COVID19). Indian J Pediatr. 2020;87:281-6. https://doi.org/10.1007/ s12098-020-03263-6.

3. World Health Organization. WHO statement regarding cluster of pneumonia cases in Wuhan, China. 2020. https://www.who.int/ china/news/detail/09-01-2020-who-statement-regarding-clusterof-pneumonia-cases-in-wuhan-china. Accessed 24 Apr 2021. 
4. World Health Organization. WHO coronavirus disease (COVID19) dashboard. 2020. https://covid19.who.int/. Accessed 06 June 2021.

5. World Health Organization. Novel coronavirus (2019-nCoV) situation report-11. 2020. https://www.who.int/docs/default-source/ coronaviruse/situation-reports/20200131-sitrep-11-ncov.pdf? sfvrsn=de7c0f7_4. Accessed 24 Apr 2021.

6. World Health Organization. Novel coronavirus (2019-nCoV) situation report-51. 2020. https://www.who.int/docs/default-source/ coronaviruse/situation-reports/20200311-sitrep-51-covid-19.pdf? sfvrsn=1ba62e57_10. Accessed 24 Apr 2021.

7. World Health Organization. COVID-19 weekly operational update on COVID-19. 2021. https://www.who.int/publications/m/item/ weekly-operational-update-on-covid-19---14-june-2021. Accessed 21 June 2021.

8. Hindson J. COVID-19: faecal-oral transmission? Nat Rev Gastroenterol Hepatol. 2020;17:259. https://doi.org/10.1038/ s41575-020-0295-7.

9. Huang C, Wang Y, Li X, Ren L, Zhao J, Hu Y, et al. Clinical features of patients infected with 2019 novel coronavirus in Wuhan, China. Lancet. 2020;395(10223):497-506. https://doi.org/10. 1016/S0140-6736(20)30183-5.

10. Tay MZ, Poh CM, Rénia L, MacAry PA, Ng LFP. The trinity of COVID-19: immunity, inflammation and intervention. Nat Rev Immunol. 2020;20:363-74. https://doi.org/10.1038/ s41577-020-0311-8.

11. Dai M, Liu D, Liu M, Zhou F, Li G, Chen Z, et al. Patients with cancer appear more vulnerable to SARS-CoV-2: a multicenter study during the COVID-19 outbreak. Cancer Discov. 2020;10(6):783-91. https://doi.org/10.1158/2159-8290. CD-20-0422.

12. Liang W, Guan W, Chen R, Wang W, Li J, Xu K, et al. Cancer patients in SARS-CoV-2 infection: a nationwide analysis in China. Lancet Oncol. 2020;21(3):335-7. https://doi.org/10.1016/S14702045(20)30096-6.

13. Desai A, Sachdeva S, Parekh T, Desai R. COVID-19 and cancer: lessons from a pooled meta analysis. JCO Glob Oncol. 2020;6:557-9. https://doi.org/10.1200/GO.20.00097.

14. Zhang L, Zhu F, Xie L, Wang C, Wang J, Chen R, et al. Clinical characteristics of COVID-19-infected cancer patients: a retrospective case study in three hospitals within Wuhan. China Ann Oncol. 2020;31(7):894-901. https://doi.org/10.1016/j.annonc.2020.03. 296.

15. Mehta V, Goel S, Kabarriti R, Cole D, Goldfinger M, AcunaVillaorduna A, et al. Case fatality rate of cancer patients with COVID-19 in a New York hospital system. Cancer Discov. 2020;10(7):935-41. https://doi.org/10.1158/2159-8290. CD-20-0516.

16. Yu J, Ouyang W, Chua MLK, Xie C. SARS-CoV-2 transmission in patients with cancer at a Tertiary Care Hospital in Wuhan, China. JAMA Oncol. 2020;6(7):1108-10. https://doi.org/10.1001/jamao ncol.2020.0980.

17. Bhattacharjee A, Patil VM, Dikshit R, Prabhash K, Singh A, Chaturvedi P. Should we wait or not? The preferable option for patients with stage IV oral cancer in COVID-19 pandemic. Head Neck. 2020;42(6):1173-8. https://doi.org/10.1002/hed.26196.

18. Wu F, Zhao S, Yu B, Chen YM, Wang W, Song ZG, et al. A new coronavirus associated with human respiratory disease in China. Nature. 2020;579(7798):265-9. https://doi.org/10.1038/ s41586-020-2008-3.

19. Sungnak W, Huang N, Bécavin C, Berg M, Queen R, Litvinukova $\mathrm{M}$, et al. SARSCoV-2 entry factors are highly expressed in nasal epithelial cells together with innate immune genes. Nat Med. 2020;26(5):681-7. https://doi.org/10.1038/s41591-020-0868-6.

20. Hoffmann M, Kleine-Weber H, Schroeder S, Krüger N, Herrler T, Erichsen S, et al. SARS-CoV-2 cell entry depends on ACE2 and
TMPRSS 2 and is blocked by a clinically proven protease inhibitor. Cell. 2020;181(2):271-280.e8. https://doi.org/10.1016/j.cell.2020. 02.052 .

21. Walls AC, Park YJ, Tortorici MA, Wall A, McGuire AT, Veesler D. Structure, function, and antigenicity of the SARS-CoV-2 spike glycoprotein. Cell. 2020;181(2):281-292.e6. https://doi.org/10. 1016/j.cell.2020.02.058.

22. Hoffmann M, Kleine-Weber H, Pöhlmann S. A multibasic cleavage site in the spike protein of SARS-CoV-2 is essential for infection of human lung cells. Mol Cell. 2020;78(4):779-784.e5. https://doi.org/10.1016/j.molcel.2020.04.022.

23. Bao L, Deng W, Huang B, Gao H, Liu J, Ren L, et al. The pathogenicity of SARS-CoV-2 in hACE2 transgenic mice. Nature. 2020;583:830-3. https://doi.org/10.1038/s41586-020-2312-y.

24. Muus C, Luecken M, Eraslan G, Waghray A, Heimberg G, Sikkema $\mathrm{L}$, et al. Integrated analyses of single-cell atlases reveal age, gender, and smoking status associations with cell type-specific expression of mediators of SARS-CoV-2 viral entry and highlights inflammatory programs in putative target cells. BioRxiv. 2020. https://doi.org/10.1101/2020.04.19.049254.

25. Sidaway P. COVID-19 and cancer: what we know so far. Nat Rev Clin Oncol. 2020;17(6):336. https://doi.org/10.1038/ s41571-020-0366-2.

26. National Cancer Institute. Age and cancer risk. https://www.cancer.gov/about-cancer/causes-prevention/risk/age. 2021. Accessed 25 May 2021

27. Pinto BG, Oliveira AE, Singh Y, Jimenez L, Gonçalves AN, Ogava RL, et al. ACE2 expression is increased in the lungs of patients with comorbidities associated with severe COVID-19. J Infect Dis. 2020;222(4):556-63. https://doi.org/10.1093/infdis/ jiaa332.

28. Smith JC, Sausville EL, Girish V, Yuan ML, Vasudevan A, John $\mathrm{KM}$, et al. Cigarette smoke exposure and inflammatory signaling increase the expression of the SARS-CoV-2 receptor ACE2 in the respiratory tract. Dev Cell. 2020;53(5):514-29. https://doi.org/10. 1016/j.devcel.2020.05.012.

29. Stopsack KH, Mucci LA, Antonarakis ES, Nelson PS, Kantoff PW. TMPRSS2 and COVID-19: serendipity or opportunity for intervention? Cancer Discov. 2020;10(6):779-82. https://doi.org/ 10.1158/2159-8290.CD-20-0451.

30. Montopoli M, Zumerle S, Vettor R, Rugge M, Zorzi M, Catapano $\mathrm{CV}$, et al. Androgen-deprivation therapies for prostate cancer and risk of infection by SARS-CoV-2: a population-based study $(\mathrm{n}=$ 4532). Ann Oncol. 2020;31(8):1040-5. https://doi.org/10.1016/j. annonc.2020.04.479.

31. Patel VG, Zhong X, Liaw B, Tremblay D, Tsao CK, Galsky MD, et al. Does androgen deprivation therapy protect against severe complications from COVID-19? Ann Oncol. 2020;31(10):141920. https://doi.org/10.1016/j.annonc.2020.06.023.

32. Saini KS, de las Heras B, de Castro J, et al. Effect of the COVID19 pandemic on cancer treatment and research. Lancet Haematol. 2020;7(6):e432-5. https://doi.org/10.1016/S2352-3026(20) 30123-X.

33. Sica A, Colombo MP, Trama A, Horn L, Garassino MC, Torri V. Immunometabolic status of COVID-19 cancer patients. Physiol Rev. 2020;100(4):1839-50. https://doi.org/10.1152/physrev. 00018.2020.

34. Merad M, Martin JC. Pathological inflammation in patients with COVID-19: a key role for monocytes and macrophages. Nat Rev Immunol. 2020;20:355-62. https://doi.org/10.1038/ s41577-020-0331-4.

35. Moore JB, June CH. Cytokine release syndrome in severe COVID19. Science. 2020;368(6490):473-4. https://doi.org/10.1126/scien ce.abb8925.

36. Bakouny Z, Hawley JE, Choueiri TK, Peters S, Rini BI, Warner JL. COVID-19 and cancer: current challenges and perspectives. 
Cancer Cell. 2020;38(5):629-46. https://doi.org/10.1016/j.ccell. 2020.09.018.

37. McGonagle D, O'Donnell JS, Sharif K, Emery P, Bridgewood C. Immune mechanisms of pulmonary intravascular coagulopathy in COVID-19 pneumonia. Lancet Rheumatol. 2020;2(7):e437-45. https://doi.org/10.1016/S2665-9913(20)30121-1.

38. Jose RJ, Manuel A. COVID-19 cytokine storm: the interplay between inflammation and coagulation. Lancet Respir Med. 2020;8(6):e46-7. https://doi.org/10.1016/S2213-2600(20) 30216-2.

39. Moll M, Zon RL, Sylvester KW, Chen EC, Cheng V, Connell NT, et al. VTE in ICU patients with COVID-19. Chest. 2020;158(5):2130-5. https://doi.org/10.1016/j.chest.2020.07.031.

40. Geisslinger F, Vollmar AM, Bartel K. Cancer patients have a higher risk regarding COVID-19 — and vice versa? Pharmaceuticals (Basel). 2020;13(7):143. https://doi.org/10.3390/ph130 70143.

41. Bhardwaj K, Liu P, Leibowitz JL, Kao CC. The coronavirus endoribonuclease Nsp15 interacts with retinoblastoma tumor suppressor protein. J Virol. 2012;86(8):4294-304. https://doi.org/10. 1128/JVI.07012-11.

42. Han HJ, Nwagwu C, Anyim O, Ekweremadu C, Kim S. COVID19 and cancer: from basic mechanisms to vaccine development using nanotechnology. Int Immunopharmacol. 2021;90: 107247. https://doi.org/10.1016/j.intimp.2020.107247.

43. Venkataraman T, Coleman C, Frieman MB. Overactive epidermal growth factor receptor signaling leads to increased fibrosis after severe acute respiratory syndrome coronavirus infection. J Virol. 2017;91:e00182-e217. https://doi.org/10.1128/JVI.00182-17.

44. Piersma B, Hayward MK, Weaver VM. Fibrosis and cancer: a strained relationship. Biochim Biophys Acta Rev Cancer. 2020;1873(2):188356. https://doi.org/10.1016/j.bbcan.2020. 188356.
45. Francescangeli F, De Angelis ML, Baiocchi M, Rossi R, Biffoni M, Zeuner A. COVID-19-induced modifications in the tumor microenvironment: do they affect cancer reawakening and metastatic relapse? Front Oncol. 2020;10:592891. https://doi.org/10. 3389/fonc.2020.592891.

46. Francescangeli F, De Angelis ML, Zeuner A. COVID-19: a potential driver of immune-mediated breast cancer recurrence? Breast Cancer Res. 2020;22:117. https://doi.org/10.1186/ s13058-020-01360-0.

47. Stewart CA, Gay CM, Ramkumar K, Cargill KR, Cardnell RJ, Nilsson MB, et al. Lung cancer models reveal SARS-CoV-2-induced EMT contributes to COVID-19 pathophysiology. bioRxiv. 2020. https://doi.org/10.1101/2020.05.28.122291.

48. Saygideger Y, Sezan A, Candevir A, Demir BS, Guzel E, Baydar O, et al. COVID-19 patients' sera induce epithelial mesenchymal transition in cancer cells. Cancer Treat Res Commun. 2021;28:100406. https://doi.org/10.1016/j.ctarc.2021.100406.

49. Abid MB, Mughal M, Abid MA. Coronavirus disease 2019 (COVID-19) and immune-engaging cancer treatment. JAMA Oncol. 2020;6(10):1529-30. https://doi.org/10.1001/jamaoncol. 2020.2367.

50. Nagar H, Formenti SC. Cancer and COVID-19-potentially deleterious effects of delaying radiotherapy. Nat Rev Clin Oncol. 2020;17:332-4. https://doi.org/10.1038/s41571-020-0375-1.

51. Spicer J, Chamberlain C, Papa S. Provision of cancer care during the COVID-19 pandemic. Nat Rev Clin Oncol. 2020;17:329-31. https://doi.org/10.1038/s41571-020-0370-6.

Publisher's Note Springer Nature remains neutral with regard to jurisdictional claims in published maps and institutional affiliations. 